MATHEMATICS OF COMPUTATION

Volume 75, Number 255, July 2006, Pages 1553-1560

S 0025-5718(06)01842-4

Article electronically published on March 21, 2006

\title{
COMPUTING THE ARITHMETIC GENUS OF HILBERT MODULAR FOURFOLDS
}

\author{
H. G. GRUNDMAN AND L. E. LIPPINCOTT
}

\begin{abstract}
The Hilbert modular fourfold determined by the totally real quartic number field $k$ is a desingularization of a natural compactification of the quotient space $\Gamma_{k} \backslash \mathcal{H}^{4}$, where $\Gamma_{k}=\operatorname{PSL}_{2}\left(\mathcal{O}_{k}\right)$ acts on $\mathcal{H}^{4}$ by fractional linear transformations via the four embeddings of $k$ into $\mathbf{R}$. The arithmetic genus, equal to one plus the dimension of the space of Hilbert modular cusp forms of weight $(2,2,2,2)$, is a birational invariant useful in the classification of these varieties. In this work, we describe an algorithm allowing for the automated computation of the arithmetic genus and give sample results.
\end{abstract}

\section{INTRODUCTION}

Let $k$ be a totally real algebraic number field of degree $n$ over $\mathbf{Q}$ with ring of integers $\mathcal{O}_{k}$ and discriminant $d_{k}$. For $i=1, \ldots, n$ and $a \in k$, let $a \mapsto a^{(i)}$ denote the $i$ th embedding of $k$ into the real numbers. The group $\mathrm{SL}_{2}\left(\mathcal{O}_{k}\right)$ acts on $\mathcal{H}^{n}$, the product of $n$ copies of the complex upper half plane, by

$$
\left(\begin{array}{ll}
a & b \\
c & d
\end{array}\right)\left(z_{1}, z_{2}, \ldots, z_{n}\right)=\left(\frac{a^{(1)} z_{1}+b^{(1)}}{c^{(1)} z_{1}+d^{(1)}}, \frac{a^{(2)} z_{2}+b^{(2)}}{c^{(2)} z_{2}+d^{(2)}}, \ldots, \frac{a^{(n)} z_{n}+b^{(n)}}{c^{(n)} z_{n}+d^{(n)}}\right) .
$$

The Hilbert modular group of the field $k$ is $\Gamma_{k}=\operatorname{PSL}_{2}\left(\mathcal{O}_{k}\right)$. The Hilbert modular variety of $k$ is a desingularization of the natural compactification of $\Gamma_{k} \backslash \mathcal{H}^{n}$. For more detailed discussions of the construction of these varieties, see [7] and [12].

The arithmetic genus, in the sense of Hirzebruch 8, is a birational invariant useful in the classification of nonsingular varieties. Specifically, it is equal to one plus the dimension of the space of Hilbert modular cusp forms of weight $(2,2,2,2)$. By [4, the arithmetic genus of the Hilbert modular variety of $k$ is given by

$$
(-1)^{n} \operatorname{vol}\left(\Gamma_{k} \backslash \mathcal{H}^{n}\right)+\sum_{\eta} E\left(\Gamma_{k}, \eta\right)+\sum_{\kappa} L\left(\Gamma_{k}, \kappa\right),
$$

where the first summation is over a complete set of representatives of the $\Gamma_{k^{-}}$ equivalence classes of fixed points of $\Gamma_{k}$ and the second is over the set of cusps. Precise definitions of $E\left(\Gamma_{k}, \eta\right)$ and $L\left(\Gamma_{k}, \kappa\right)$ are given below.

Hirzebruch [7] provided a formula for computing the arithmetic genus in the cases where the ring $\mathcal{O}_{k}$ contains a unit of norm -1 . This has been widely used for computing the arithmetic genus of Hilbert modular varieties of odd dimension, since -1 is a unit of norm -1 in any field of odd degree. For varieties of even

Received by the editor April 23, 2004 and, in revised form, May 10, 2005.

2000 Mathematics Subject Classification. Primary 11F41, 14E08; Secondary 14J10, 14J35.

The first author wishes to acknowledge the support of the Faculty Research Fund of Bryn Mawr College.

(c)2006 American Mathematical Society Reverts to public domain 28 years from publication 1553 
dimension, the computation is more complicated. In the 1970's, Hirzebruch, van de Ven, and Zagier [9, 10] demonstrated how to carry out these computations for surfaces, but until recently little progress was made with higher degrees. It must be said that part of this is due to the earlier lack of computational tools. For example, a challenge as little as a decade ago, the computation of the volume term, $(-1)^{n} \operatorname{vol}\left(\Gamma_{k} \backslash \mathcal{H}^{n}\right)$, is all but trivialized by now-available software packages.

In [6], the current authors focused on the degree four case and determined how to compute the fixed point term, $\sum_{\eta} E\left(\Gamma_{k}, \eta\right)$. This left the cusp term, $\sum_{\kappa} L\left(\Gamma_{k}, \kappa\right)$, as the final roadblock to completing the computations. In this paper, we offer a now complete solution to the problem, providing an algorithm for computing the arithmetic genus of the Hilbert modular variety of an arbitrary quartic field $k$, and describing our implementation thereof.

We develop the necessary mathematics and describe our algorithm for computing the cusp term in Section 2. In Section 3 we review the needed results from [6] and describe our algorithms for computing the volume and fixed point terms. Finally, in Section 4, we describe our specific implementation and give a table of the arithmetic genera of the Hilbert modular fourfolds determined by the 210 totally real quartic fields of smallest discriminant.

\section{THE CUSP TERM}

In this section, we develop the tools needed for computing the cusp term,

$$
\sum_{\kappa} L\left(\Gamma_{k}, \kappa\right)
$$

for an arbitrary quartic field, $k$. We begin by defining notation.

Let $\mathcal{C}_{k}$ denote the group of ideal classes and $\mathcal{E}_{k}$ the group of units of $\mathcal{O}_{k}$. It is well known that the set of cusps of $\Gamma_{k} \backslash \mathcal{H}^{4}$ is in one-to-one correspondence with $\mathcal{C}_{k}$. Letting $[\mathfrak{a}]$ be the ideal class corresponding to the cusp $\kappa$, we have

$$
L\left(\Gamma_{k},[\mathfrak{a}]\right)=L\left(\Gamma_{k}, \kappa\right)=\frac{1}{16 \pi^{4}} \sqrt{d\left(\mathfrak{a}^{-2}\right)} \sum_{a \in \mathfrak{a}^{-2} / \mathcal{E}_{k}^{2}} \frac{\operatorname{sign}(N(a))}{|N(a)|},
$$

with $d\left(\mathfrak{a}^{-2}\right)$ the discriminant of $\mathfrak{a}^{-2}$ [4. In this notation, the cusp term is given by $\sum_{[\mathfrak{a}] \in \mathcal{C}_{k}} L\left(\Gamma_{k},[\mathfrak{a}]\right)$.

We begin with a lemma proved in [6].

Lemma 1. If $\mathcal{O}_{k}$ contains a unit of norm -1 or an integral ideal $\mathfrak{b}$ for which $\mathfrak{b}^{2}=(\beta)$ is a principal ideal with $N(\beta)<0$, then $\sum_{\kappa} L\left(\Gamma_{k}, \kappa\right)=0$.

If the hypothesis of Lemma 1 is not satisfied by $k$, then there exists a real character, $\psi: \mathcal{I}_{k} \rightarrow \pm 1$, on the group of nonzero fractional ideals, satisfying $\psi((a))=\operatorname{sign}(N(a))$ for all nonzero $a \in \mathcal{O}_{k}$. It follows that $\bar{\psi}\left(\mathfrak{a}^{2}\right)=1$ and so, letting $x$ vary over integral ideals in $\mathcal{O}_{k}$,

$$
\begin{aligned}
\sum_{[\mathfrak{a}] \in \mathcal{C}_{k}} L\left(\Gamma_{k},[\mathfrak{a}]\right) & =\frac{\sqrt{d_{k}}}{\pi^{4}} \lim _{s \rightarrow 1} \sum_{[\mathfrak{a}] \in \mathcal{C}_{k}} \sum_{x \in\left[\mathfrak{a}^{2}\right]} \frac{\psi(x)}{N(x)^{s}} \\
& =\frac{\sqrt{d_{k}}}{\pi^{4}} \lim _{s \rightarrow 1} \sum_{[x] \in \mathcal{C}_{k}^{2}} \frac{\psi(x)}{N(x)^{s}}\left|\mathcal{C}_{k} / \mathcal{C}_{k}^{2}\right|
\end{aligned}
$$


As in [6], let $\Phi$ be the set of characters $\phi: \mathcal{I}_{k} \rightarrow \pm 1$ induced by a complete set of characters on the group $\mathcal{C}_{k} / \mathcal{C}_{k}^{2}$. Then,

$$
\begin{aligned}
\sum_{[\mathfrak{a}] \in \mathcal{C}_{k}} L\left(\Gamma_{k},[\mathfrak{a}]\right) & =\frac{\sqrt{d_{k}}}{\pi^{4}} \lim _{s \rightarrow 1} \sum_{x} \frac{\psi(x)}{N(x)^{s}} \sum_{\phi \in \Phi} \phi(x) \\
& =\frac{\sqrt{d_{k}}}{\pi^{4}} \sum_{\phi \in \Phi} \lim _{s \rightarrow 1} \sum_{x} \frac{\psi \phi(x)}{N(x)^{s}} \\
& =\frac{\sqrt{d_{k}}}{\pi^{4}} \sum_{\phi \in \Phi} L(1, \psi \phi) .
\end{aligned}
$$

Now, since each $\phi$ is trivial on principal ideals, each $\psi \phi$ is a quadratic character on $\mathcal{I}_{k}$ satisfying $\psi \phi((a))=\operatorname{sign}(N(a))$ for all nonzero $a \in \mathcal{O}_{k}$. Hence, each $\psi \phi$ corresponds, via class field theory, to a quadratic extension $k^{\prime}$ of $k$, unramified at each finite place and ramified at each infinite place. In fact, as $\phi$ ranges through $\Phi$, the $k^{\prime}$ range through all such field extensions of $k$.

Since each $k^{\prime}$ is a totally complex quadratic extension of the totally real number field $k$, we have

$$
L(1, \psi \phi)=L\left(1, k^{\prime} / k\right)=\frac{\pi^{4} h_{k^{\prime}} R_{k^{\prime}} w_{k} \sqrt{d_{k}}}{h_{k} R_{k} w_{k^{\prime}} \sqrt{d_{k^{\prime}}}}
$$

where $h_{k}, h_{k^{\prime}}, R_{k}, R_{k^{\prime}}, d_{k}, d_{k^{\prime}}$ are the class numbers, regulators, and absolute values of the discriminants of $\mathcal{O}_{k}$ and $\mathcal{O}_{k^{\prime}}$, respectively, and $w_{k}, w_{k^{\prime}}$ are the numbers of roots of unity in these rings.

Combining the above with the fact that $w_{k}=2$ yields the following theorem.

Theorem 2. If $\mathcal{O}_{k}$ contains a unit of norm -1 or an integral ideal $\mathfrak{b}$ for which $\mathfrak{b}^{2}=(\beta)$ is a principal ideal with $N(\beta)<0$, then $\sum_{\kappa} L\left(\Gamma_{k}, \kappa\right)=0$. Otherwise,

$$
\sum_{\kappa} L\left(\Gamma_{k}, \kappa\right)=\frac{2}{h_{k} R_{k}} \sum_{k^{\prime}} \frac{h_{k^{\prime}} R_{k^{\prime}}}{w_{k^{\prime}}}
$$

where $k^{\prime}$ ranges through the set of unramified, totally complex, quadratic extensions of $k$.

To compute the sum in equation (2), we use the computer algebra package KASH [3. We determine the set of quadratic fields, $k^{\prime}$, as follows. RayClassField and RayClassFieldAuto are used to determine the narrow class field of $k$ (that is, the field corresponding to the group of positive principal divisors). Then OrderSubfield is used to find all degree 8 subfields. OrderSig is used to determine which of these subfields are totally complex, and OrderIsSubfield is used to determine which of the totally complex subfields contain $k$. Once the appropriate quadratic extensions of $k$ have been determined, the commands OrderClassGroup, OrderReg, and OrderTorsionUnitRank can be used to evaluate each summand.

This computation is carried out only when $\mathcal{O}_{k}$ contains no unit of norm -1 and no integral ideal $\mathfrak{b}$ for which $\mathfrak{b}^{2}=(\beta)$ is a principal ideal with $N(\beta)<0$. We check for a unit of norm -1 by using the commands OrderUnitsFund and EltNorm. If none exists, we determine whether there is an integral ideal $\mathfrak{b}$ for which $\mathfrak{b}^{2}=(\beta)$ is a principal ideal with $N(\beta)<0$, by using OrderClassGroupCyclicFactors. Viewing the class group as a product of cyclic groups, this command provides, for each cyclic factor, the order of the factor and an ideal in a class that generates it. 
For each cyclic factor of even order, we raise the representative ideal to the order of the factor, thus producing a principal ideal. Finally, we use IdealIsPrincipal to obtain a generator for the ideal and EltNorm to determine whether the norm of the ideal's generator is negative.

If any of these generators is of negative norm, then setting it equal to $\beta$ gives us that by Theorem 2. $\sum_{\kappa} L\left(\Gamma_{k}, \kappa\right)=0$. We now show that if no such generator exists, then no such $\beta$ exists.

Lemma 3. If the above process fails to yield a generator of negative norm, then every generator of any principal ideal equal to the square of an integral ideal in $\mathcal{O}_{k}$ has positive norm.

Proof. Suppose that $\mathfrak{b}$ is an integral ideal such that $\mathfrak{b}^{2}=(\beta)$ and $N(\beta)<0$. Since $k$ is totally real and contains no unit of norm $-1, \mathfrak{b}$ is not principal and so is in an ideal class of order 2 .

Let $\mathfrak{c}_{1}, \mathfrak{c}_{2}, \ldots, \mathfrak{c}_{n}$ be representative ideals for generators for the cyclic factors of the class group of $k$. Let $e_{1}, e_{2}, \ldots, e_{n}$ be minimal nonnegative integers such that $\mathfrak{c}_{1}^{e_{1}} \mathfrak{c}_{2}^{e_{2}} \cdots \mathfrak{c}_{n}^{e_{n}}$ is in the same ideal class as $\mathfrak{b}$. So we have

$$
\alpha \mathfrak{c}_{1}^{e_{1}} \mathfrak{c}_{2}^{e_{2}} \cdots \mathfrak{c}_{n}^{e_{n}}=\gamma \mathfrak{b},
$$

for some $\alpha, \gamma \in \mathcal{O}_{k}$. Thus

$$
\alpha^{2} \mathfrak{c}_{1}^{2 e_{1}} \mathfrak{c}_{2}^{2 e_{2}} \cdots \mathfrak{c}_{n}^{2 e_{n}}=\gamma^{2}(\beta) .
$$

Since each side of equation (3) is a principal ideal, the hypothesis gives us that each $\mathfrak{c}_{i}^{2 e_{i}}$ has a generator of positive norm. (Note that $e_{i}=0$ for any cyclic factor of the class group of odd order, since each $\mathfrak{c}_{i}^{2 e_{i}}$ is principal.) Hence $\gamma^{2}(\beta)$ and therefore $(\beta)$ have generators of positive norm. Since $\mathcal{O}_{k}$ has no units of norm -1 , all generators of $(\beta)$ have positive norms. This contradicts our supposition and concludes the proof.

\section{The VOlume AND FIXed POINT TERMS}

As noted in [5], $\operatorname{vol}\left(\Gamma_{k} \backslash \mathcal{H}^{n}\right)=\frac{1}{8} \zeta_{k}(-1)$, which is easily computed using the command zetak in the software package PARI 1]. It follows from the work of Siegel [11, 13] that $15 \zeta_{k}(-1)$ is a rational integer. Although PARI does not provide specific error-bounds for zetak, all of the outputs were within $10^{-25}$ of an integral multiple of $\frac{1}{15}$.

Computing the fixed point term is more involved. As defined in [4, letting $\eta$ be the equivalence class of a fixed point $z$, this term is given by

$$
E\left(\Gamma_{k},[z]\right)=\frac{1}{\left|\Gamma_{z}\right|} \sum_{\substack{M \in \Gamma_{z} \\ M \neq I}} \prod_{i=1}^{4}\left(\frac{1}{1-\zeta_{i}}\right),
$$

where $\Gamma_{z}$ is the isotropy subgroup of $z$ and $\zeta$ is the rotation factor of $\bar{M}$. We summarize our computational formula for this term in the following theorem, which is easily derived from [6, Theorem 4.2].

For any field $k^{\prime}$, let $h_{k^{\prime}}$ and $R_{k^{\prime}}$ be the class number and regulator of $\mathcal{O}_{k^{\prime}}$ respectively. For $r \in\{2,3,4,5,6\}$ let

$$
m_{r}=\left(R_{k\left(e^{\frac{\pi i}{r}}\right)} h_{k\left(e^{\frac{\pi i}{r}}\right)}\right) /\left(R_{k} h_{k}\right),
$$

and let $\delta$ be the relative discriminant of $k\left(e^{\frac{\pi i}{r}}\right)$ over $k$. 
Theorem 4. Let $k$ be a totally real quartic number field.

If $d_{k}=1125$, then $\sum_{\eta} E\left(\Gamma_{k}, \eta\right)=\frac{17}{10}$.

If $d_{k}=2000$, then $\sum_{\eta} E\left(\Gamma_{k}, \eta\right)=\frac{151}{60}$.

If $d_{k}=2048$, then $\sum_{\eta} E\left(\Gamma_{k}, \eta\right)=\frac{91}{48}$.

If $d_{k}=2304$, then $\sum_{\eta} E\left(\Gamma_{k}, \eta\right)=\frac{61}{24}$.

If $d_{k} \notin\{1125,2000,2048,2304\}$, then

$$
\sum_{\eta} E\left(\Gamma_{k}, \eta\right)=\frac{m_{2}}{96} f_{2}+\frac{m_{3}}{216} f_{3}+\frac{m_{4}}{64} f_{4}+\frac{m_{5}}{40} f_{5}+\frac{m_{6}}{864} f_{6},
$$

where the $f_{r}$ are as given in Table 1 .

The following corollary simplifies the computation.

TABLE 1 . Values of $f_{r}$ for $r=2,3,4,5,6$

\begin{tabular}{|ccc|rrr|}
\multicolumn{7}{c}{$\sqrt{2} \in k, \sqrt{3} \notin k$} \\
\hline $2 \mathcal{O}_{k}$ & $2 \mathcal{O}_{k^{\prime}}$ & $\delta$ & $f_{2}$ & $f_{4}$ & $f_{6}$ \\
\hline $\mathfrak{p}^{2}$ & - & - & 6 & 3 & 0 \\
$\mathfrak{p}^{2} \mathfrak{q}^{2}$ & - & - & 12 & 3 & 0 \\
$\mathfrak{p}^{4}$ & $\mathfrak{s}^{4}$ & - & 54 & 38 & 0 \\
$\mathfrak{p}^{4}$ & $\mathfrak{s}^{4} \mathfrak{t}^{4}$ & - & 18 & 20 & 0 \\
$\mathfrak{p}^{4}$ & $\mathfrak{s}^{8}$ & - & 18 & 9 & 0 \\
\hline
\end{tabular}

\begin{tabular}{|ccc|ccc|}
\multicolumn{2}{c}{$\sqrt{2} \notin k, \sqrt{3} \in k$} \\
\hline $2 \mathcal{O}_{k}$ & $2 \mathcal{O}_{k^{\prime}}$ & $\delta$ & $f_{2}$ & $f_{4}$ & $f_{6}$ \\
\hline $\mathfrak{p}^{2}$ & $\mathfrak{s}^{2}$ & - & 25 & 0 & 209 \\
$\mathfrak{p}^{2}$ & $\mathfrak{s}^{2} \mathfrak{t}^{2}$ & - & 15 & 0 & 209 \\
$\mathfrak{p}^{2} \mathfrak{q}^{2}$ & - & - & 99 & 0 & 209 \\
$\mathfrak{p}^{4}$ & - & - & 45 & 0 & 209 \\
\hline
\end{tabular}

\begin{tabular}{|ccc|rrr|}
\multicolumn{7}{c}{$\sqrt{2} \notin k, \sqrt{3} \notin k$} \\
\hline $2 \mathcal{O}_{k}$ & $2 \mathcal{O}_{k^{\prime}}$ & $\delta$ & $f_{2}$ & $f_{4}$ & $f_{6}$ \\
\hline $\mathfrak{p}$ & - & - & 3 & 0 & 0 \\
$\mathfrak{p q}$ & - & - & 3 & 0 & 0 \\
$\mathfrak{p q r}$ & - & - & 3 & 0 & 0 \\
$\mathfrak{p q r} \mathfrak{r}$ & - & - & 3 & 0 & 0 \\
$\mathfrak{p}^{2}$ & $\mathfrak{s}^{2}$ & - & 78 & 0 & 0 \\
$\mathfrak{p}^{2}$ & $\mathfrak{s}^{2} \mathfrak{t}^{2}$ & - & 48 & 0 & 0 \\
$\mathfrak{p}^{2}$ & $\mathfrak{s}^{4}$ & - & 15 & 0 & 0 \\
$\mathfrak{p}^{2} \mathfrak{q}$ & $\mathfrak{s}^{2} \mathfrak{t}^{2}$ & - & 30 & 0 & 0 \\
$\mathfrak{p}^{2} \mathfrak{q}$ & $\mathfrak{s}^{2} \mathfrak{t}^{2} \mathfrak{u}^{2}$ & - & 12 & 0 & 0 \\
$\mathfrak{p}^{2} \mathfrak{q}$ & $\mathfrak{s}^{4} \mathfrak{t}^{2}$ & - & 9 & 0 & 0 \\
$\mathfrak{p}^{2} \mathfrak{q r}$ & $\mathfrak{s}^{2} \mathfrak{t}^{2} \mathfrak{u}^{2}$ & - & 30 & 0 & 0 \\
$\mathfrak{p}^{2} \mathfrak{q} \mathfrak{r}$ & $\mathfrak{s}^{2} \mathfrak{t}^{2} \mathfrak{u}^{2} \mathfrak{v}^{2}$ & - & 12 & 0 & 0 \\
$\mathfrak{p}^{2} \mathfrak{q} \mathfrak{r}$ & $\mathfrak{s}^{4} \mathfrak{t}^{2} \mathfrak{u}^{2}$ & - & 9 & 0 & 0 \\
$\mathfrak{p}^{2} \mathfrak{q}^{2}$ & $\mathfrak{s}^{2} \mathfrak{t}^{2}$ & - & 300 & 0 & 0 \\
$\mathfrak{p}^{2} \mathfrak{q}^{2}$ & $\mathfrak{s}^{2} \mathfrak{t}^{2} \mathfrak{u}^{2}$ & - & 120 & 0 & 0 \\
$\mathfrak{p}^{2} \mathfrak{q}^{2}$ & $\mathfrak{s}^{2} \mathfrak{t}^{2} \mathfrak{u}^{2} \mathfrak{v}^{2}$ & - & 48 & 0 & 0 \\
$\mathfrak{p}^{2} \mathfrak{q}^{2}$ & $\mathfrak{s}^{4} \mathfrak{t}^{2}$ & - & 90 & 0 & 0 \\
$\mathfrak{p}^{2} \mathfrak{q}^{2}$ & $\mathfrak{s}^{4} \mathfrak{t}^{2} \mathfrak{u}^{2}$ & - & 36 & 0 & 0 \\
$\mathfrak{p}^{2} \mathfrak{q}^{2}$ & $\mathfrak{s}^{4} \mathfrak{t}^{4}$ & - & 27 & 0 & 0 \\
$\mathfrak{p}^{3} \mathfrak{q}$ & $\mathfrak{s}^{\mathfrak{t}^{2}}$ & $\mathfrak{u}^{2} \mathfrak{v}^{2}$ & 21 & 0 & 0 \\
$\mathfrak{p}^{3} \mathfrak{q}$ & $\mathfrak{s}^{6} \mathfrak{t}^{2}$ & $\mathfrak{u}^{4} \mathfrak{v}^{2}$ & 9 & 0 & 0 \\
$\mathfrak{p}^{4}$ & $\mathfrak{s}^{4}$ & - & 138 & 0 & 0 \\
$\mathfrak{p}^{4}$ & $\mathfrak{s}^{4} \mathfrak{t}^{4}$ & - & 48 & 0 & 0 \\
$\mathfrak{p}^{4}$ & $\mathfrak{s}^{8}$ & $\mathfrak{u}^{2}$ & 45 & 0 & 0 \\
$\mathfrak{p}^{4}$ & $\mathfrak{s}^{8}$ & $\mathfrak{u}^{4}$ & 21 & 0 & 0 \\
\hline
\end{tabular}

\begin{tabular}{|c|c|c|}
\hline \multicolumn{3}{|c|}{$\sqrt{3} \notin k$} \\
\hline $3 \mathcal{O}_{k}$ & $3 \mathcal{O}_{k^{\prime}}$ & $f_{3}$ \\
\hline $\begin{array}{l}\mathfrak{p} \\
\end{array}$ & - & 9 \\
\hline $\mathfrak{p q}$ & - & 9 \\
\hline $\mathfrak{p q r}$ & - & 9 \\
\hline $\mathfrak{p q} \mathfrak{r} \mathfrak{s}$ & & 9 \\
\hline $\mathfrak{p}^{2}$ & $\mathfrak{s}^{2}$ & 54 \\
\hline $\mathfrak{p}^{2}$ & $\mathfrak{s}^{2} \mathfrak{t}^{2}$ & 90 \\
\hline $\mathfrak{p}^{2} \mathfrak{q}$ & $\mathfrak{s}^{2} \mathfrak{t}^{2}$ & 45 \\
\hline $\mathfrak{p}^{2} \mathfrak{q}$ & $\mathfrak{s}^{2} \mathfrak{t}^{2} \mathfrak{u}^{2}$ & 27 \\
\hline $\mathfrak{p}^{2} \mathfrak{q} \mathfrak{r}$ & $\mathfrak{s}^{2} \mathfrak{t}^{2} \mathfrak{u}^{2}$ & 45 \\
\hline $\mathfrak{p}^{2} \mathfrak{q} \mathfrak{r}$ & $\mathfrak{s}^{2} \mathfrak{t}^{2} \mathfrak{u}^{2} \mathfrak{v}^{2}$ & 27 \\
\hline $\mathfrak{p}^{2} \mathfrak{q}^{2}$ & $\mathfrak{s}^{2} \mathfrak{t}^{2}$ & 234 \\
\hline $\mathfrak{p}^{2} \mathfrak{q}^{2}$ & $\mathfrak{s}^{2} \mathfrak{t}^{2} \mathfrak{u}^{2}$ & 144 \\
\hline $\mathfrak{p}^{2} \mathfrak{q}^{2}$ & $\mathfrak{s}^{2} \mathfrak{t}^{2} \mathfrak{u}^{2} \mathfrak{v}^{2}$ & 90 \\
\hline $\mathfrak{p}^{3} \mathfrak{q}$ & 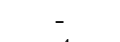 & 36 \\
\hline $\mathfrak{p}^{4}$ & $\mathfrak{s}^{4}$ & 162 \\
\hline $\mathfrak{p}^{4}$ & $\mathfrak{s}^{4} \mathfrak{t}^{4}$ & 90 \\
\hline \multicolumn{3}{|c|}{$\sqrt{3} \in k$} \\
\hline $3 \mathcal{O}_{k}$ & $3 \mathcal{O}_{k^{\prime}}$ & $f_{3}$ \\
\hline $\mathfrak{p}^{2}$ & & 40 \\
\hline $\mathfrak{p}^{2} \mathfrak{q}^{2}$ & & 112 \\
\hline $\mathfrak{p}^{4}$ & & 76 \\
\hline
\end{tabular}
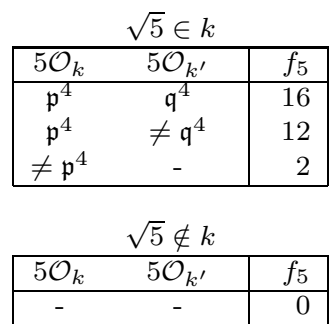
Corollary 5. Let $k$ be a totally real quartic field.

If $\sqrt{2} \notin k$, then $f_{4}=0$.

If $\sqrt{5} \notin k$, then $f_{5}=0$.

If $\sqrt{3} \notin k$, then $f_{6}=0$.

To compute the values of $\sum_{\eta} E\left(\Gamma_{k}, \eta\right)$ we first use the KASH function OrderDisc to check if $k$ is any of the four special fields listed in Theorem 4, If not, we evaluate equation (5) as follows: EltRoot determines which, if any, of $\sqrt{2}, \sqrt{5}$, and $\sqrt{3}$ are in $k$; then applying Corollary 5 as appropriate determines the values of some of the $f_{r}$. For the remaining $f_{r}$, we use Table 1 to determine what ideals need to be factored and use OrderDisc to define any needed relative discriminants. The function Factor is used to factor the ideals, and the exponents of the factorization are extracted for use with the table. The values of the $m_{r}$ are computed directly using the KASH functions OrderReg and OrderClassGroup for the regulators and class numbers, respectively.

TABLE 2. Arithmetic genera of Hilbert modular fourfolds

\begin{tabular}{|c|c|c|c|c|c|c|c|c|c|c|c|}
\hline$d_{k}$ & $\chi$ & $d_{k}$ & $\chi$ & $d_{k}$ & $\chi$ & $d_{k}$ & $\chi$ & $d_{k}$ & $\chi$ & $d_{k}$ & $\chi$ \\
\hline 725 & 1 & 7625 & 5 & 13068 & 7 & 17609 & 5 & 22221 & 7 & 26125 & 12 \\
\hline 1125 & 2 & 8000 & 5 & 13448 & 6 & 17725 & 7 & 22545 & 9 & 26176 & 10 \\
\hline 1600 & 2 & 8069 & 3 & 13525 & 6 & 17989 & 5 & 22592 & 9 & 26224 & 9 \\
\hline 1957 & 1 & 8112 & 4 & 13625 & 7 & 18097 & 7 & 22676 & 9 & 26225 & 10 \\
\hline 2000 & 3 & 8468 & 3 & 13676 & 6 & 18432 & 8 & 22784 & 11 & 26525 & 10 \\
\hline 2048 & 2 & 8525 & 4 & 13725 & 7 & 18496 & 14 & 22896 & 8 & 26541 & 9 \\
\hline 2225 & 2 & 8725 & 4 & 13768 & 5 & 18625 & 9 & 23252 & 8 & 26569 & 12 \\
\hline 2304 & 3 & 8768 & 4 & 13824 & 7 & 18688 & 10 & 23297 & 7 & 26825 & 9 \\
\hline 2525 & 2 & 8789 & 3 & 13888 & 6 & 18736 & 6 & 23301 & 9 & 26873 & 8 \\
\hline 2624 & 2 & 8957 & 3 & 13968 & 9 & 19025 & 8 & 23377 & 7 & 27004 & 10 \\
\hline 2777 & 1 & 9225 & 5 & 14013 & 5 & 19225 & 8 & 23525 & 9 & 27225 & 14 \\
\hline 3600 & 4 & 9248 & 5 & 14197 & 5 & 19429 & 6 & 23552 & 9 & 27329 & 8 \\
\hline 3981 & 2 & 9301 & 3 & 14272 & 5 & 19525 & 8 & 23600 & 9 & 27472 & 9 \\
\hline 4205 & 2 & 9792 & 5 & 14336 & 6 & 19600 & 10 & 23665 & 7 & 27648 & 13 \\
\hline 4225 & 3 & 9909 & 5 & 14400 & 8 & 19664 & 12 & 23724 & 11 & 27725 & 10 \\
\hline 4352 & 4 & 10025 & 5 & 14656 & 7 & 19773 & 10 & 24197 & 9 & 27792 & 14 \\
\hline 4400 & 3 & 10273 & 3 & 14725 & 6 & 19796 & 12 & 24336 & 13 & 28025 & 11 \\
\hline 4525 & 3 & 10304 & 5 & 15125 & 8 & 19821 & 7 & 24400 & 12 & 28224 & 13 \\
\hline 4752 & 4 & 10309 & 4 & 15188 & 5 & 20032 & 8 & 24417 & 9 & 28224 & 14 \\
\hline 4913 & 3 & 10512 & 7 & 15317 & 5 & 20225 & 8 & 24437 & 8 & 28400 & 11 \\
\hline 5125 & 4 & 10816 & 5 & 15529 & 5 & 20308 & 7 & 24525 & 11 & 28473 & 9 \\
\hline 5225 & 3 & 10889 & 3 & 15952 & 5 & 20808 & 9 & 24749 & 7 & 28669 & 9 \\
\hline 5725 & 3 & 11025 & 7 & 16225 & 7 & 21025 & 9 & 24832 & 15 & 28677 & 9 \\
\hline 5744 & 3 & 11197 & 3 & 16317 & 7 & 21056 & 8 & 24917 & 8 & 28749 & 10 \\
\hline 6125 & 4 & 11324 & 5 & 16357 & 5 & 21200 & 8 & 25088 & 11 & 29237 & 8 \\
\hline 6224 & 3 & 11344 & 4 & 16400 & 9 & 21208 & 7 & 25225 & 10 & 29248 & 11 \\
\hline 6809 & 2 & 11348 & 4 & 16448 & 7 & 21308 & 8 & 25488 & 14 & 29268 & 13 \\
\hline 7053 & 3 & 11525 & 5 & 16448 & 7 & 21312 & 10 & 25492 & 9 & 29813 & 9 \\
\hline 7056 & 5 & 11661 & 4 & 16609 & 5 & 21469 & 7 & 25525 & 10 & 29952 & 13 \\
\hline 7168 & 4 & 12197 & 4 & 16997 & 6 & 21568 & 11 & 25717 & 7 & 30056 & 18 \\
\hline 7225 & 4 & 12357 & 6 & 17069 & 5 & 21725 & 8 & 25808 & 9 & 30056 & 16 \\
\hline 7232 & 4 & 12400 & 6 & 17417 & 6 & 21737 & 8 & 25857 & 14 & 30125 & 14 \\
\hline 7488 & 5 & 12544 & 9 & 17424 & 10 & 21801 & 9 & 25893 & 8 & 30273 & 10 \\
\hline 7537 & 2 & 12725 & 5 & 17428 & 6 & 21964 & 10 & 25961 & 8 & 30400 & 12 \\
\hline 7600 & 4 & 13025 & 6 & 17600 & 7 & 22000 & 11 & 26032 & 9 & 30512 & 11 \\
\hline
\end{tabular}




\section{IMPLEMENTATION AND RESULTS}

Our implementation consists of a shell script calling two programs: a simple PARI program that produces a table of values of $\zeta_{k}(-1)$, and a KASH program that performs the remainder of the calculations. As input, the script takes a file of fields in a form output by PARI as found at the University of Bordeaux's number fields web site 2, with one field per line. Our PARI program creates a new file of $\zeta_{k}(-1)$ values which our KASH program reads along with the original input file. For each field in the input file, the KASH program computes the volume term, the fixed point term, and the cusp term as described in this paper and combines them to compute the arithmetic genus.

We ran the programs using PARI 2.1.4 and KASH 2.2 on a SUN Ultra-60, with a $450 \mathrm{MHz}$ Sun UltraSPARC-II processor, running the Solaris 5.8 operating system. Results for the Hilbert modular varieties defined by the 210 totally real quartic fields of smallest discriminant are given in Table 2, As an indication of the time involved in these calculations, it takes approximately 40 minutes to compute the first 100 cases, 100 minutes to compute the 210 given in the table, and 13 hours to compute the first 1000 .

As a final note, running the program using KASH 2.4 resulted in errors, apparently arising from a problem with the OrderSubfield command.

\section{REFERENCES}

[1] C. Batut, K. Belabas, D. Benardi, H. Cohen, and M. Olivier. User's Guide to PARI-GP, 1998. 〈ftp://megrez.math.u-bordeaux.fr/pub/pari $\rangle$.

[2] J. Buchmann, F. Diaz y Diaz, D. Ford, P. Létard, M. Olivier, M. Pohst, and A. Schwarz. Tables of number fields of low degree, 〈ftp://megrez.math.u-bordeaux.fr/pub/ numberfields/ $\rangle$.

[3] M. Daberkow, C. Fieker, J. Klüners, M. Pohst, K. Roegner, and K. Wildanger. Kant v4. J. Symbolic Comp., 24:267-283, 1997. 〈http://www.math.TU-Berlin.DE/ kant/kash.html〉. MR.1484479 (99g:11150)

[4] E. Freitag. Hilbert Modular Forms. Springer-Verlag, Berlin, 1980. MR1050763 (91c:11025)

[5] H. G. Grundman. Hilbert modular varieties over Galois quartic fields. J. Number Theory, 63 (1997), 47-58. MR1438648 (98c:11040)

[6] H. G. Grundman and L. E. Lippincott. Hilbert modular fourfolds of arithmetic genus one. In High Primes and Misdemeanours: lectures in honour of the 60th birthday of Hugh Cowie Williams, Fields Inst. Commun., 41, pp. 217-226. Amer, Math. Soc., 2004. MR2076248 (2005d:11065)

[7] F. Hirzebruch. Hilbert modular surfaces. Enseign. Math., II. Ser., 19 (1973), 183-281. MR0393045 (52:13856)

[8] F. Hirzebruch. Topological Methods in Algebraic Geometry. Springer-Verlag, Berlin, 3rd edition, 1978. MR.1335917 (96c:57002)

[9] F. Hirzebruch and A. Van de Ven. Hilbert modular surfaces and the classification of algebraic surfaces. Invent. Math., 23 (1974), 1-29. MR0364262 (51:517)

[10] F. Hirzebruch and D. Zagier. Classification of Hilbert modular surfaces. In Complex Analysis and Algebraic Geometry, pp. 43-77. University Press and Iwanami Shoten, Cambridge, 1977. MR0480356 (58:524)

[11] C. L. Siegel. Berechnung von Zetafunktionen an ganzzahligen Stellen. Nachr. Akad. Wiss. Göttingen II. Math. Phys. Kl., pages 87-102, 1969. MR0252349(40:5570)

[12] G. van de Geer. Hilbert Modular Surfaces. Springer-Verlag, Berlin, 1988. MR0930101 (89c:11073)

[13] D. Zagier. On the values at negative integers of the zeta-function of a real quadratic field. Enseign. Math, 22 (1976), 55-95. MR0406957 (53:10742) 
Bryn Mawr College, 101 N. Merion Ave., Bryn Mawr, Pennsylvania 19010

E-mail address: grundman@brynmawr.edu

Bryn Mawr College, 101 N. Merion Ave., Bryn Mawr, Pennsylvania 19010

E-mail address: llippinc@brynmawr.edu 